\title{
Time-Domain Simulation of Electronic Noises
}

\author{
Alberto Pullia and Stefano Riboldi
}

\begin{abstract}
In this paper, a procedure is proposed to computer simulate the electronic noise of ionizing-radiation spectrometers. The viewpoint of the simulator is the output of the preamplifier, with or without an anti-aliasing filter, just in front of the ADC. Examples are given for the case of segmented high purity Germanium detectors (HPGe). The method makes use of the fractional calculus basics. A software procedure provides the noisy waveform as a function of the fundamental electrical-physical parameters of the system, including: detector capacitance, detector leakage current, feedback resistor, $1 / f$-noise coefficient of the input transistor, temperature of the preamplifier input devices.o The ADC quantization noise is also included in the simulation.
\end{abstract}

Index Terms-Electronic noise, ionizing radiation detectors, noise simulation.

\section{INTRODUCTION}

$\mathbf{T}$ HE new generation of cylindrical HPGe detectors for $\gamma$-ray spectroscopy is characterized by 25 - to 36 -fold segmentation of the electrode located on the outer cylindrical-hexagonal surface of the crystal [1], [2]. Segmentation is needed to enhance the detector granularity and permits to estimate the incidence angle of the gamma photon trajectories. The three-dimensional (3-D) coordinates of the interaction points of the $\gamma$-photons inside the detector can be estimated with an accuracy of a few mm's through sophisticated algorithms of pulse shape analysis (PSA). Such algorithms take into account both the shape of the charge signal seen on the collecting electrode and that seen on the adjacent segments (induced charge signals). Currently the best results have been obtained using genetic or neural algorithms, which (i) require a training phase, (ii) are nonlinear, and (iii) are mostly developed in the time domain [3]. In order to test such algorithms efforts have been made to simulate the charge signals seen at the segment electrodes versus the position of the interaction points, considering different geometries of the detector and the electrodes [4], [5], but little or no effort has been made to simulate the fundamental electronic noise superimposed to such signals, i.e. the shot noise of the detector leakage current, the thermal, $1 / f$, and Lorentzian noises of the preamplifier input transistor, the thermal noise of the feedback resistor. This work is intended to fill such a gap. It hopefully opens the way to a complete simulation of the noisy detector signals and should permit to get more realistic figures about the effectiveness of the PSA algorithms. Even if other approaches are possible, we believe

Manuscript received November 14, 2003; revised May 4, 2004. This work was supported by the Italian Istituto Nazionale di Fisica Nucleare (INFN) and Ministero dell'Istruzione, dell'Università e della Ricerca (MIUR).

A. Pullia is with the Department of Physics, University of Milano, and INFN, 20133 Milano, Italy (e-mail: alberto.pullia@mi.infn.it).

S. Riboldi is with the Department of Electronics, Politecnico of Milano, and INFN, 20133 Milano, Italy (e-mail: riboldi@elet.polimi.it).

Digital Object Identifier 10.1109/TNS.2004.832564 that the simplest way to study the effect of the electronic noise on so-complex algorithms consists of simply adding it and then run suitable tests. A computer program has been written in the matlab environment, that provides a noisy waveform referred to the preamplifier output versus a pattern of fundamental electrical-physical parameters of the system.

\section{THEORETICAL BACKGROUND}

The noise of a detector-preamplifier setup for X-and $\gamma$-ray spectroscopy is always colored. As referred to the detector input current, its power spectral density includes typically $(i)$ a white component, (ii) a component proportional to $|f|$, being $f$ the frequency, and (iii) a component proportional to $|f|^{2}$ [6]. Other components, such as Lorentzian-noise packets or higher order $|f|^{\alpha}(\alpha>2)$ components, can be present. Such a noise pattern propagates through the preamplifier transfer function and appears after such a "shaping" network at the preamplifier output, or the observation point of our context. One has to consider that the charge preamplifier has a finite bandwidth or a finite intrinsic risetime. It can hence be modeled by a transfer function with one pole in the origin (integration of the detector current upon its feedback capacitance) and a pole due to its finite risetime, typically 10 to $40 \mathrm{~ns}$. All of the noise components (i), (ii), and (iii) can be referred to the preamplifier output by multiplying their power spectra by the squared absolute value of such a transfer function. Input noise components $(i)$, (ii), and (iii) can be modeled in the time domain in terms of a Poissonian sequence of given core pulses of fixed amplitude and shape but random sign. However a computer representation of such core pulses is not possible because they contain $\delta$-Dirac-like components or, stricly speaking, they are not functions but distributions. A further complication arises because the $1 / f$-noise representation is obtained through $1 / 2$-order integration [7]-[9] of $\delta$-Dirac pulses. However this is only a practical complication and poses no conceptual limitation to a straightforward development of the model. It is worth noting that the spectral containt of all $\delta$-Dirac-like core pulses keeps finite when the frequency is pushed to infinity, whereas no known physical phenomenon is such. The difficulty to translate $\delta$-Dirac-like components into a computer representation can be removed if they are made band-limited by the transfer function of a physical system. Note that in this framework the physical system is the preamplifier itself. The physical reasons why the native noise should itself be of band-limited nature could be investigated. However we can observe that the band limit of the charge-preamplifier transfer function is surely at a lower frequency than other fundamental and quanto-mechanical phenomena limiting the input noise band. So we can reasonably affirm that the noise waveform obtained by filtering the mathematical model of the input noise through the preamplifier transfer function is a realistic representation of the noise actually seen at the preamplifier output. 


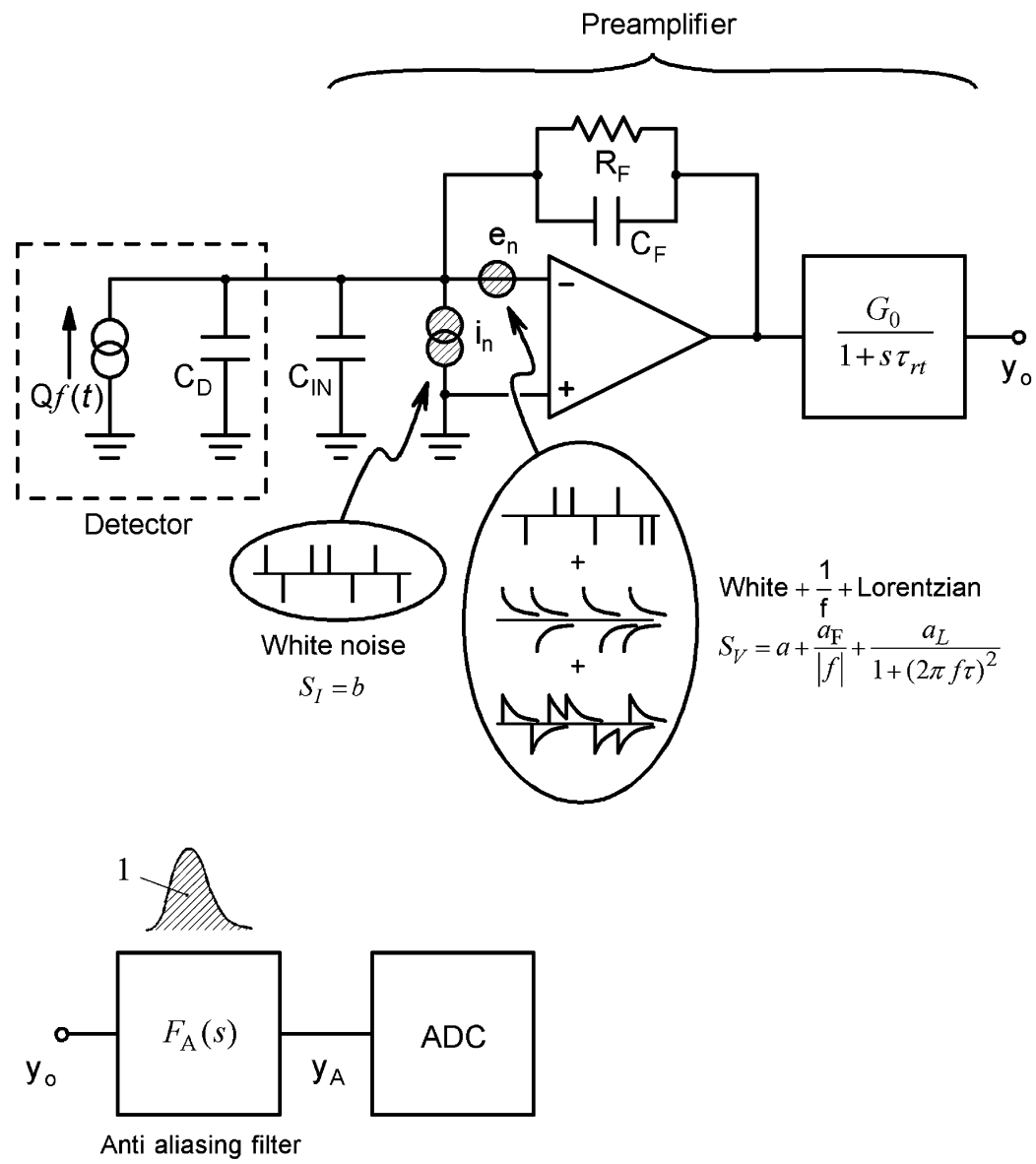

Fig. 1. Detector-preamplifier-ADC chain. The anti-aliasing filter interposed between the preamplifier and the ADC is modeled as an $n$-pole transfer function.

\section{METHOD}

Fig. 1 shows the simplified schematic diagram of a typical detector-preamplifier-ADC setup. The detector delivers current signals of the form $\mathrm{Q} f(t)$ being $\mathrm{Q}$ the charge collected at its output electrode and $f(t)$ a unit-area function modeling the charge induction transient. [4] The operational amplifier is assumed ideal apart from the noise and, in conjunction with $C_{F}$, is configured as a charge amplifier. Resistor $R_{F}$ is used to continuously reset the charge accumulated onto $\mathrm{C}_{\mathrm{F}}$. The finite bandwidth of the charge preamplifier is fictitiously introduced by means of a functional block containing a pole with time constant $\tau_{r t}\left(\tau_{r t}=\right.$ preamplifier risetime), which is a good approximation for stable systems with feedback pole smaller than $1 / 4$ of the gain-bandwidth product. In the event the stability condition is no longer true, the system response would show an overshoot and the functional block should be changed inherently. However, we will not consider this case for the sake of simplicity. Eventually a functional block with unit-area impulse response is used to model the antialiasing filter. The gain $G_{0}$ shown in Fig. 1 can be conveniently used as a normalization term, making the following cases:

$$
\begin{aligned}
G_{0} & =1 \\
G_{0} & =C_{F} \\
G_{0} & =\frac{C_{F}}{q} \psi
\end{aligned}
$$

where $q$ is the electronic charge and $\Psi$ [eV/pair] is the energy/charge conversion coefficient of the detector (e.g. $\Psi=$ $2.92 \mathrm{eV} /$ pair for germanium and $\Psi=3.6 \mathrm{eV} /$ pair for silicon). In case (1) the reading of waveforms $y_{\mathrm{o}}(t)$ or $y_{\mathrm{A}}(t)$ is in volts, in case (2) in coulombs, in case (3) in eV.

The principal input-referred noise sources $\left(e_{\mathrm{n}}, i_{\mathrm{n}}\right)$ are put into evidence in Fig. 1 and a picture of their mathematical models [8] is also shown. The used mathematical model for the white noise components is a Poissonian sequence of $\delta$-like pulses, each having area $A$ and random sign, occurring at an average rate $\nu$. It can be shown (Appendix I) that the (two-sided) power spectral density of such a noisy waveform is

$$
S_{\mathrm{W}}=\nu A^{2} .
$$

Note that (4) does not depend on frequency, as expected for a white noise. From (4) it can be seen that, given the (two-sided) spectral density of a white noise $\left(S_{\mathrm{W}}\right)$ and an arbitrary average rate of incoming pulses $(\nu)$, one can derive the area $A$ of each pulse, or

$$
A=\sqrt{\frac{S_{\mathrm{W}}}{\nu}} .
$$

The model for the $1 / f$ noise is a little more complicated. To visualize it we have to use a bit of mathematics to write conveniently its two-sided power spectral density $S_{1 / \mathrm{f}}$, or

$$
S_{\frac{1}{\mathrm{f}}}=\frac{a_{\mathrm{F}}}{|f|}=a_{\mathrm{F}}\left|\frac{1}{\sqrt{j f}}\right|^{2}=2 \pi a_{\mathrm{F}}\left|\frac{1}{\sqrt{j \omega}}\right|^{2}
$$


where $j$ is the imaginary unit, $f$ is frequency, $\omega$ is angular frequency, $a_{\mathrm{F}}$ does not depend on frequency. Noise density (6) may be obtained by passing a fictitious white noise with spectral density $S_{\mathrm{W}}=2 \pi a_{\mathrm{F}}$ through a circuit with transfer function $F=1 / \sqrt{j \omega}$. The fictitious white noise can be modeled, as stated before, as a Poisson sequence of $\delta$-like pulses occurring at an arbitrary rate $\nu_{1 / \mathrm{f}}$, each of which has random sign and area $A$ given by (5), or

$$
A=\sqrt{\frac{2 \pi a_{\mathrm{F}}}{\nu_{\frac{1}{\mathrm{f}}}}} .
$$

Each of such $\delta$ pulses passes through transfer function $F$, which corresponds in the time domain to a mathematical linear operator called " $1 / 2$-order integration", normally written with the symbol d $\mathrm{d}^{-1 / 2} / \mathrm{dt}^{-1 / 2} .1 / 2$-order integral of a $\delta$ pulse centered at $t=t_{\mathrm{k}}$ and with area $A$ has been solved in literature, or

$$
\frac{d^{-\frac{1}{2}}}{d t^{-\frac{1}{2}}}\left[A \delta\left(t-t_{k}\right)\right]=\left\{\begin{array}{ll}
\frac{A}{\sqrt{\pi\left(t-t_{k}\right)}} & t>t_{k} \\
0 & t \leq t_{k}
\end{array} .\right.
$$

Putting (7) into (8) we eventually obtain the core pulse of the $1 / f$ noise, or

$$
y_{k}(t)=\left\{\begin{array}{ll}
\sqrt{\frac{2 a_{\mathrm{F}}}{\nu_{\frac{1}{\mathrm{f}}\left(t-t_{k}\right)}}} & t>t_{k} \\
0 & t \leq t_{k}
\end{array} .\right.
$$

Our model of the $1 / f$ noise consists of a sequence of core pulses $\pm y_{k}(t)$ (the sign is random) occurring Poissonianly at times $t_{k}$ with an average rate $\nu_{1 / \mathrm{f}}$, as also sketched in Fig. 1 .

The model for the Lorentzian noise is pretty similar. The twosided power spectral density $S_{\mathrm{L}}$ of a Lorentzian noise is

$$
S_{\mathrm{L}}=\frac{a_{\mathrm{L}}}{1+\omega^{2} \tau^{2}}=a_{\mathrm{L}}\left|\frac{1}{1+j \omega \tau}\right|^{2} \text {. }
$$

Noise density (10) may again be obtained by passing a fictitious white noise with spectral density $S_{\mathrm{W}}=a_{\mathrm{L}}$ through a circuit with transfer function $F=1 /(1+j \omega \tau)$, i.e. a passive $R C$ network having time constant $\tau$. It is worth recalling that the $R C$ network is an imperfect integrator, which makes this case pretty similar to the previous one. Again the fictitious white noise consists of a Poisson sequence of $\delta$ pulses occurring at an arbitrary rate $\nu_{\mathrm{L}}$, each of which has random sign and area $A_{\mathrm{L}}$ given by (5), or

$$
A_{\mathrm{L}}=\sqrt{\frac{a_{\mathrm{L}}}{\nu_{\mathrm{L}}}} .
$$

Each of such $\delta$ pulses gets "integrated" by the RC network and appears at its output in the form

$$
y_{m}(t)= \begin{cases}\sqrt{\frac{a_{\mathrm{L}}}{\nu_{\mathrm{L}}}} \frac{e^{-\frac{t-t_{m}}{\tau}}}{\tau} & t>t_{m} \\ 0 & t \leq t_{m}\end{cases}
$$

being $t_{m}$ the arrival time of the corresponding $\delta$ pulse. So, our model of the Lorentzian noise consists of a sequence of core pulses $\pm y_{m}(t)$ occurring Poissonianly at times $t_{m}$ with an average rate $\nu_{\mathrm{L}}$, as also sketched in Fig. 1.

In the next Sections we will determine more precisely the amplitude of the core pulses of the input noises vs the key electrical-physical parameters of the system and propagate them to the preamplifier output.

\section{INPUT NOISE CORE PULSES}

Input noises $e_{n}$ and $i_{n}$ are uncorrelated and have the following (two-sided) power spectral densities [10]:

$$
\begin{aligned}
& S_{V}=\frac{d \overline{e_{n}^{2}}}{d f}=a+\frac{a_{\mathrm{F}}}{|f|}+\frac{a_{\mathrm{L}}}{1+(2 \pi f \tau)^{2}} \\
& S_{I}=\frac{d \overline{i_{n}^{2}}}{d f}=b
\end{aligned}
$$

where parameters $a, a_{\mathrm{F}}, a_{\mathrm{L}}, \tau$ and $b$ do not depend on frequency. " $a$ " is the white component of the series noise of the preamplifier input transistor. " $b$ " includes the shot noises of the detector leakage current and the preamplifier input bias current plus the thermal noise of the reset resistor. Namely,

$$
\begin{aligned}
a & =2 K T R_{S} \\
b & =q I_{L}+\frac{2 K T}{R_{F}}
\end{aligned}
$$

where $K$ is the Boltzmann constant, $T$ is absolute temperature. For an input FET $R_{S}=\alpha / g_{\mathrm{m}}$ where $g_{\mathrm{m}}$ is its transconductance and $\alpha$ is a constant factor $\approx 2 / 3$ [11], and for an input BJT $R_{S}=1 / 2 g_{\mathrm{m}}+R_{\mathrm{bb}^{\prime}}$ where $R_{\mathrm{bb}^{\prime}}$ is the base spreading resistor. $I_{L}$ is the sum of the detector leakage current and the input transistor bias current (the latter is typically negligible for an FET cooled to cryogenic temperatures), $R_{F}$ is the feedback resistor. $a_{\mathrm{L}}$ and $\tau$ are the amplitude and time parameters of an individual Lorentzian (or trapping) noise source. We do not consider the case of a packet of Lorentzian noise sources for the sake of simplicity. Substituting (15) and (16) in (13) and (14), and using (5) we derive the area

$$
A_{\mathrm{S}}=\sqrt{\frac{2 K T R_{S}}{\nu_{\mathrm{S}}}}
$$

for the series white noise core pulses, and the area

$$
A_{\mathrm{P}}=\sqrt{\frac{q I_{L}+\frac{2 K T}{R_{F}}}{\nu_{\mathrm{P}}}}
$$

for the parallel white noise core pulses. $A_{\mathrm{S}}$ and $A_{\mathrm{P}}$ are the areas of the $\delta$ pulses that we shall use to correctly model the series and parallel white noises. The noisy waveforms $e_{n}$ and $i_{n}$ are built as Poissonian sequences of such pulses, each pulse taken with random sign.

In Table I, the core pulses of all considered noise components are shown in a mathematical form. It is worth pointing out that the average rates of incoming pulses $\nu_{\mathrm{S}}, \nu_{\mathrm{P}}, \nu_{1 / \mathrm{f}}, \nu_{\mathrm{L}}$ i.e. the occurrence rate of each of the Poisson time sequences $t_{h}, t_{l}, t_{k}$, $t_{m}$, can be chosen arbitrarily. Note, however, that they appear in the relations of Table I into terms $A_{\mathrm{S}}, A_{\mathrm{P}}, A, A_{\mathrm{L}}$ as shown by (17), (18), (7), (11), and always in the denominator. In fact the lower the rate of incoming pulses, the higher the weight of each noise pulse (and viceversa) to keep the noise intensity constant.

\section{OutPut-Noise Core Pulses}

We now want to propagate the input noise waveforms to the preamplifier output. Let us begin with the series noise $e_{n}$. It is easily found that in the circuit of Fig. 1 the frequency domain relationship

$$
y_{0}=e_{n} \frac{1+s R_{F} C_{T}}{1+s \tau_{F}} \frac{G_{0}}{1+s \tau_{r t}}
$$


TABLE I

CORE PULSES OF NOISE COMPONENTS

\begin{tabular}{|c|c|c|c|c|}
\hline $\begin{array}{l}\text { Noise } \\
\text { component }\end{array}$ & $\begin{array}{l}\text { Label } \\
\text { as }\end{array}$ & \multicolumn{2}{|c|}{$\begin{array}{l}\text { Core pulse* } \\
\text { (time domain) }\end{array}$} & $\begin{array}{l}\text { Core pulse** } \\
\text { (freq. domain) }\end{array}$ \\
\hline $\begin{array}{l}\text { Series } \\
\text { white }\end{array}$ & $\begin{array}{c}y_{h} \\
(h=1,2, \ldots)\end{array}$ & \multicolumn{2}{|c|}{$A_{\mathrm{S}} \delta\left(t-t_{h}\right)$} & $A_{\mathrm{S}} e^{-s t_{h}}$ \\
\hline $\begin{array}{l}\text { Parallel } \\
\text { white }\end{array}$ & $\begin{array}{c}y_{l} \\
(l=1,2, \ldots)\end{array}$ & \multicolumn{2}{|c|}{$A_{\mathrm{P}} \delta\left(t-t_{l}\right)$} & $A_{\mathrm{P}} e^{-s t_{l}}$ \\
\hline $\begin{array}{l}\text { Series } \\
1 / f\end{array}$ & $\begin{array}{c}y_{k} \\
(k=1,2, \ldots)\end{array}$ & $\frac{A}{\sqrt{\pi\left(t-t_{k}\right)}} \begin{array}{c}0 \\
0\end{array}$ & $\begin{array}{l}t>t_{k} \\
t \leq t_{k}\end{array}$ & $\frac{A}{\sqrt{s}} e^{-s t_{k}}$ \\
\hline $\begin{array}{l}\text { Series } \\
\text { Lorentzian }\end{array}$ & $\begin{array}{c}y_{m} \\
(m=1,2, \ldots)\end{array}$ & $\left\{\begin{array}{c}A_{\mathrm{L}} \frac{e^{-\frac{t-t_{m}}{\tau}}}{\tau} \\
0\end{array}\right.$ & $\begin{array}{l}t>t_{m} \\
t \leq t_{m}\end{array}$ & $\frac{A_{\mathrm{L}}}{1+s \tau} e^{-s t_{m}}$ \\
\hline
\end{tabular}

* $A_{\mathrm{S}}, A_{\mathrm{P}}, A, A_{\mathrm{L}}$ are given respectively by (17), (18), (7) and (11)

$* *$ in this column assume $s=j \omega$

holds, where $s=j \omega, C_{T}=C_{D}+C_{I N}+C_{F}$ and $\tau_{F}=$ $R_{F} C_{F} \cdot \tau_{F}$ is typically in the range from $200 \mu \mathrm{s}$ to $2 \mathrm{~ms}$, and is very large compared to the processing time of the signals (both in pulse-shape analysis and in pulse-height analysis), which is typically in the range between $200 \mathrm{~ns}$ and $20 \mu \mathrm{s}$. So, in (19) we can make the approximation

$$
\tau_{F} \rightarrow \infty
$$

and, being $R_{F} C_{T}>\tau_{F}$

$$
R_{F} C_{T} \rightarrow \infty .
$$

Substituting (20) and (21) in (19) we obtain

$$
y_{\mathrm{ser}}^{\mathrm{o}} \approx e_{n} \frac{C_{T}}{C_{F}} \frac{G_{0}}{1+s \tau_{r t}}
$$

and, after the anti-aliasing filter

$$
y_{\text {ser }}^{\mathrm{A}}=y_{\text {ser }}^{0} F_{\mathrm{A}}(s)
$$

being $F_{\mathrm{A}}(s)$ the transfer function of the antialiasing filter.

Using a similar procedure for the parallel noise $i_{n}$ again looking at the circuit of Fig. 1, we obtain the relationship

$$
y_{\mathrm{par}}^{\mathrm{o}} \approx i_{n} \frac{1}{s C_{F}} \frac{G_{0}}{1+s \tau_{r t}}
$$

and, after the anti-aliasing filter,

$$
y_{\text {par }}^{\mathrm{A}}=y_{\text {par }}^{0} F_{\mathrm{A}}(s) .
$$

We have now to find the time domain counterparts of (22), (23), (24) and (25). We can do this for single input core pulses (of the noisy waveforms $e_{n}$ and $i_{n}$ ) centered at $t=0$ (Table I with $t_{h}=t_{l}=t_{k}=t_{m}=0$ ).

\section{A. Series White Noise}

With these assumptions, putting a core pulse of the series white noise centered at $t=0$ (see first row, right column of Table I with $t_{h}=0$ ) into (22) in place of $e_{n}$ and solving the in- verse Fourier transform one obtains the time domain core pulse of the series white noise as seen at the preamplifier output, or

$$
\underset{\text { ser }}{y \circ}(t)= \begin{cases}A_{\mathrm{S}} \frac{C_{T}}{C_{F}} \frac{G_{0}}{\tau_{r t}} e^{-\frac{t}{\tau_{r t}}}, & t \geq 0 \\ 0, & t<0\end{cases}
$$

where $A_{\mathrm{S}}$ is given by (17) and and $G_{0}$ is given by (1), (2) or (3), depending on whether the reading is in volts, coulombs or $\mathrm{eV}$. Note that in cases (2) and (3) the dependence of $y_{0}(t)$ on $C_{F}$ gets canceled out. In fact in these cases $y_{0}(t)$ is normalized in terms of detector charge or event energy, which by no means depend on $C_{F}$.

Using (23) and (26) one easily obtains the time domain core pulse of the series white noise as seen at the anti-aliasing filter output, or

$$
y_{\mathrm{ser}}^{\mathrm{A}}(t)=y_{\mathrm{ser}}^{0}(t) * f_{\mathrm{A}}(t)
$$

where $f_{\mathrm{A}}(t)$ is the unit-area impulse response of the anti aliasing filter and the star stands for time convolution. Time convolution (27) could be solved in closed form for simple antialiasing filters but in practice it is convenient to solve it numerically for any simple or complex $f_{\mathrm{A}}(t)$.

\section{B. Parallel White Noise}

Putting a core pulse of the parallel white noise centered at $t=0$ (see second row, right column of Table I with $t_{l}=0$ ) into (24) in place of $i_{n}$ and solving the inverse Fourier transform one obtains the time domain core pulse of the parallel white noise as seen at the preamplifier output, or

$$
\underset{\text { par }}{y \text { o }}= \begin{cases}A_{\mathrm{P}} \frac{G_{0}}{C_{F}}\left(1-e^{-\frac{t}{\tau_{r t}}}\right), & t \geq 0 \\ 0, & t<0\end{cases}
$$

where $A_{\mathrm{P}}$ is given by (18) and and $G_{0}$ is given by (1), (2) or (3). From (25) and (28) one obtains the time domain core pulse of the parallel white noise as seen at the anti-aliasing filter output, or

$$
\underset{\text { par }}{y_{\mathrm{p}}^{\mathrm{A}}}=\underset{\mathrm{par}}{0} * f_{\mathrm{A}}(t) .
$$

Time convolution (29) can be again solved numerically for any $f_{\mathrm{A}}(t)$.

\section{Series $1 / f$ Noise}

Putting a core pulse of the series $1 / f$ noise centered at $t=0$ (see third row, right column of Table I with $t_{k}=0$ ) into (22) in place of $e_{n}$ and solving the inverse Fourier transform one obtains the time domain core pulse of the series $1 / f$ noise as seen at the preamplifier output, or

$$
y_{\frac{1}{f}}= \begin{cases}A \frac{C_{T}}{C_{F}} \frac{G_{0}}{\sqrt{2 \pi \tau_{r t}}}\left|e^{-\frac{t}{\tau_{r t}}} \operatorname{erf}\left(j \sqrt{\frac{t}{\tau_{r t}}}\right)\right|, & t \geq 0 \\ 0, & t<0\end{cases}
$$

where $\operatorname{erf}(z)$ is the well known error function for real values of $z$, or its analytical continuation for complex values of $z$. A is given by (7), $G_{0}$ is given by (1), (2), or (3).

Using (20) and (30) one can now obtain the time domain core pulse of the series $1 / f$ noise as seen at the anti-aliasing filter output, or

$$
y_{\substack{\mathrm{A} \\ \frac{1}{f}}}=y_{\frac{1}{f}} * f_{A}(t) .
$$




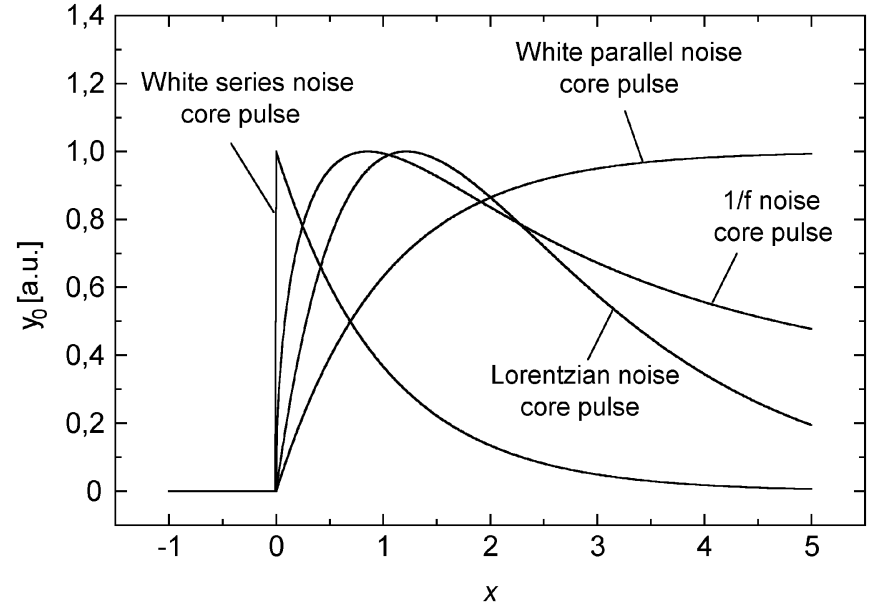

Fig. 2. Core pulses (27), (29), and (31) of the series, parallel and $1 / f$ noises as seen at the preamplifier output and plotted vs normalized time $x=t / \tau_{r t}$.

Time convolution (31) can be again solved numerically for any $f_{\mathrm{A}}(t)$.

\section{Series Lorentzian (Trapping) Noise}

Putting a core pulse of the series Lorentzian noise centered at $t=0$ (see fourth row, right column of Table I with $t_{m}=0$ ) into (22) in place of $e_{n}$ and solving the inverse Fourier transform one obtains the time domain core pulse of the series Lorentzian noise as seen at the preamplifier output, or

$$
y_{\text {Lor }}^{\circ}(t)=\left\{\begin{array}{ll}
A_{\mathrm{L}} \frac{C_{T}}{C_{F}} G_{0} \frac{e^{-\frac{t}{\tau_{r t}}}-e^{-\frac{t}{\tau}}}{\frac{1}{\tau}-\frac{1}{\tau_{r t}}}, & t \geq 0 \\
0, & t<0
\end{array} .\right.
$$

Equation (32) holds if $\tau \neq \tau_{\mathrm{rt}}$. The probability that $\tau_{\mathrm{rt}}=\tau$ is very low. However it can be shown that in such case (32) becomes

$$
y_{\text {Lor }}^{\circ}(t)=\left\{\begin{array}{ll}
A_{\mathrm{L}} \frac{C_{T}}{C_{F}} G_{0} t e^{-\frac{t}{\tau}}, & t \geq 0 \\
0, & t<0
\end{array} .\right.
$$

From (25) and (32) one obtains the time domain core pulse of the parallel white noise as seen at the anti-aliasing filter output, or

$$
y_{\text {Lor }}^{\mathrm{A}}=y_{\text {Lor }}^{0} * f_{A}(t) .
$$

Time convolution (34) can be again solved numerically for any $f_{\mathrm{A}}(t)$.

In Fig. 2 the noise core pulses seen at the preamplifier output, i.e. (26), (28) and (30), (32) are graphically shown. Note that the preamplifier transforms the input noise core pulses into plain functions with no $\delta$-like components or local divergencies.

\section{EXAMPLE}

A simple software procedure has been written, that builds a noisy waveform (referred to the preamplifier output) as described in the previous Sections. The input parameters of the procedure are: $T$ (absolute temperature), $R_{S}$ (input-transistor noise resistor), $R_{F}$ (feedback resistor), $I_{L}$ (detector leakage current), $C_{T}$ (total capacitance at the preamplifier input), $a_{F}$ $(1 / f$-noise two-sided power spectral density at $1 \mathrm{~Hz})$. The
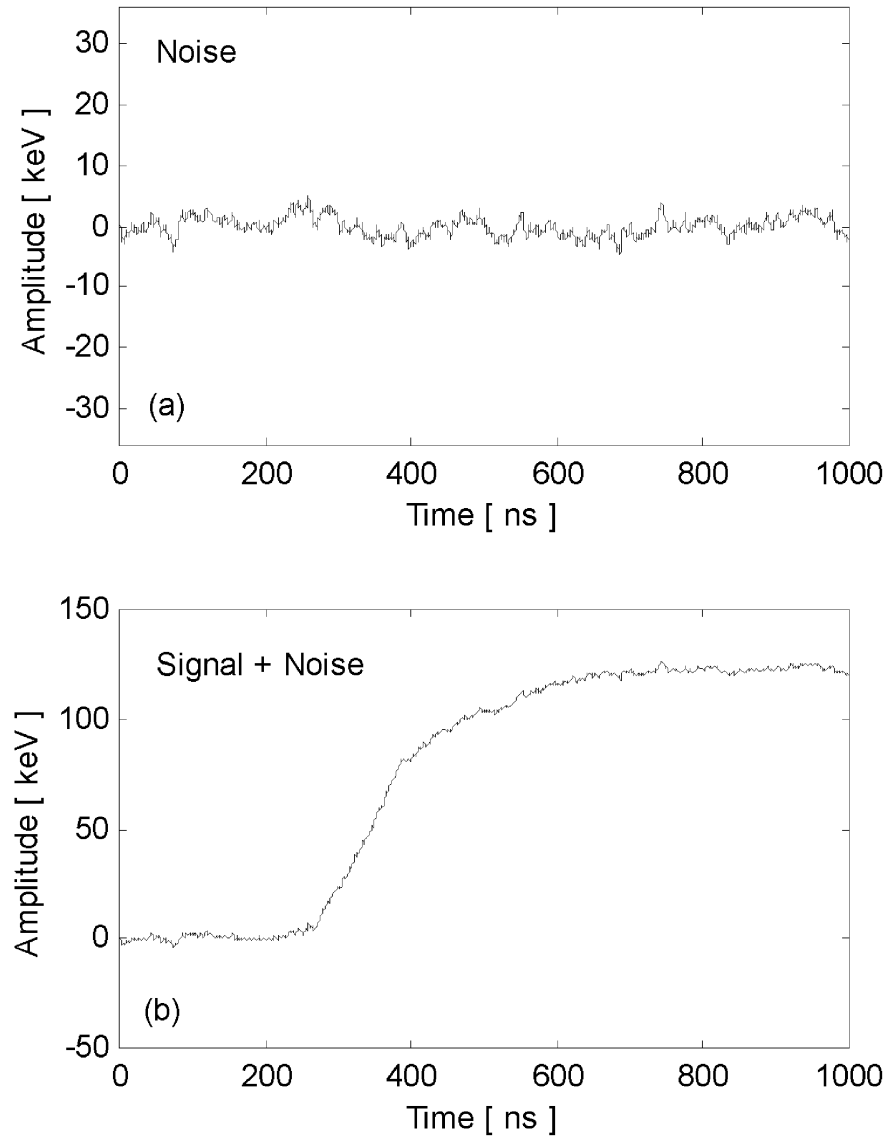

Fig. 3. In graph (a) a simulation of the noise is depicted, where the electrical-physical parameters shown in the text are used. In picture (b) the noise is superimposed to the signal (122 keV event) simulated for a truly coaxial germanium detector (inner radius $=0.5 \mathrm{~cm}$, outer radius $=3 \mathrm{~cm}$, interaction - point radius $=0.9 \mathrm{~cm}$ ).

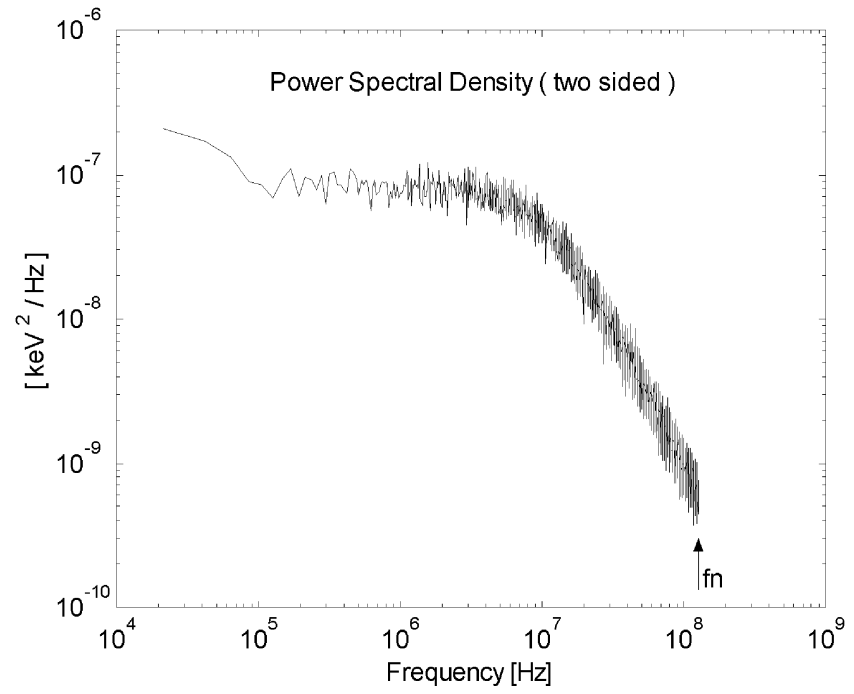

Fig. 4. Power spectral density (two sided) of the simulated noise shown in Fig. 3. The $-40 \mathrm{~dB} / \mathrm{dec}$ drop at high frequencies is due to the finite bandwidth of the preamplifier $\left(\tau_{r t}\right)$. fn is $1 / 4$ of the Nyquist frequency.

noise provided by the software procedure is in terms of charge (coulomb) or in terms of energy $(\mathrm{eV})$. In this latter case the energy-to-charge conversion factor of the detector must be specified. In Figs. 3 and 4 an example is given, with the typical 


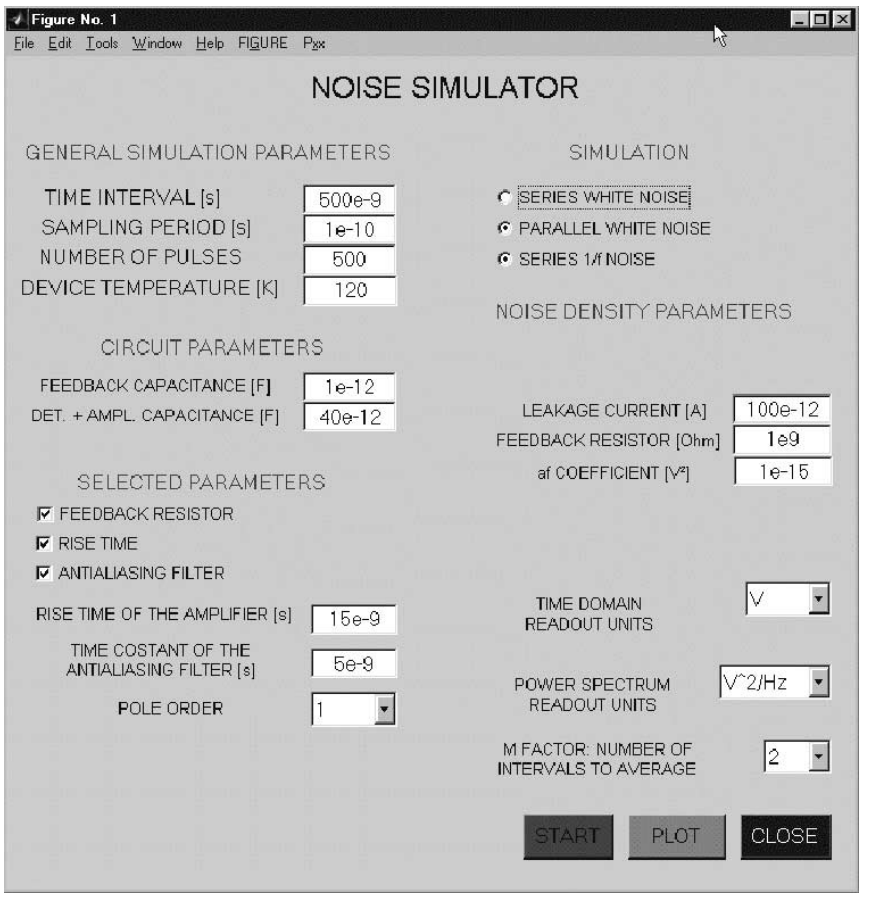

Fig. 5. A picture of the realized graphical user interface. The physical system parameters as well as the preferred readout units are easily introduced.

parameters of an HPGe segmented detector, or $\mathrm{T}=120 \mathrm{~K}$, $g_{\mathrm{m}}=15 \mathrm{mS}, R_{F}=1 \mathrm{G} \Omega, I_{L}=100 \mathrm{pA}, C_{T}=40 \mathrm{pF}$, $a_{F}=1 \times 10^{-15} \mathrm{~V}^{2}, \tau_{\mathrm{rt}}=15 \mathrm{~ns}$. No anti-aliasing filter is included $(n=0)$. A picture of the realized graphical user interface (GUI) is shown in Fig. 5.

\section{EXPERIMENTAL VALIDATION}

Accurate estimation of model parameters is mandatory in order to generate truthful time domain simulations of noise signals from HPGe detectors. Because of the unavoidable discrepancies between the supposed and the actual values of system parameters, e.g. preamplifier and detector capacitance, FET trans-conductance, etc. realistic noise simulations for a given measurement set-up can be obtained only by acquiring a large collection of noise pulses and subsequently tuning the noise simulator model to fit the experimental data.

In order to prove the effectiveness of the adopted noise simulation model in a realistic condition, experimental signals have been acquired from the MARS coaxial HPGe detector $(90 \mathrm{~mm}$ long, with outer radius $=36 \mathrm{~mm}$ and inner radius $=$ $5 \mathrm{~mm}$ ) and the resulting noise power spectral density function has been plotted in Fig. 6. Note that the experimental noise density function is consistent with the one obtained from the simulated noise (see Fig. 4), apart from a slight difference in noise level. That is because the actual noise parameters describing the experimental signals: $\mathrm{T}=120 \mathrm{~K}, g_{\mathrm{m}}=13 \mathrm{mS}$, $R_{F}=1 \mathrm{G} \Omega, I_{L}=110 \mathrm{pA}, C_{T}=49 \mathrm{pF}, a_{F}=1 \times 10^{-15}$, $\tau_{\mathrm{rt}}=13 \mathrm{~ns}$, derived from a fitting procedure (see Fig. 6) are somehow different from the first guess values used for the noise simulator.

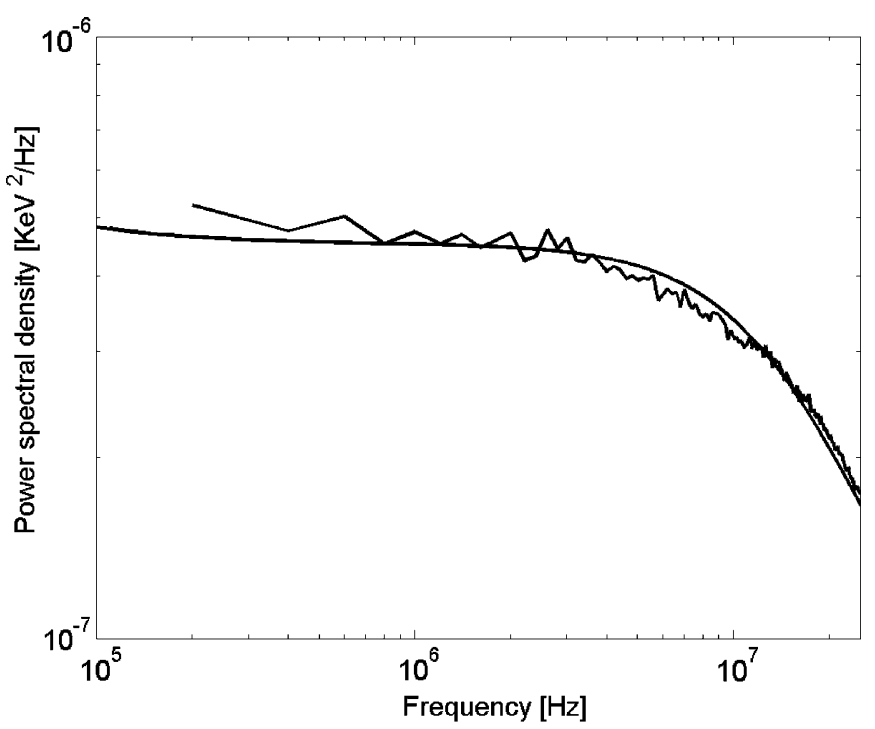

Fig. 6. Noise power spectral density function calculated from the acquired signals (jagged curve) and the corresponding fitting function as a function of noise parameters (smooth curve). The lower frequency limit is imposed by the finite acquisition time for the experimental signals, while the upper boundary has been set to $1 / 4$ of the Nyquist frequency to avoid aliasing effects.

\section{APPENDIX I}

Let us transmit a Poissonian sequence of $\delta$ pulses, each having area $A$ and random sign, through an RC network (assume $\mathrm{RC}=\tau$ ), i.e. a linear system with transfer function

$$
F=\frac{1}{1+j \omega \tau} .
$$

Each input $\delta$ impulse appears at the output as an exponential decay function with decay time constant $\tau$, or

$$
\begin{cases} \pm A \frac{e^{-\frac{t}{\tau}}}{\tau}, & t>0 \\ 0, & t \leq 0\end{cases}
$$

where the sign is random.

The output process is a Poissonian sequence of pulses of the type (36). The variance $\sigma^{2}$ of such a process can be obtained using the Campbell's theorem, or

$$
\sigma^{2}=\nu \int_{0}^{\infty}\left(A \frac{e^{-\frac{t}{\tau}}}{\tau}\right)^{2} d t=\frac{\nu A^{2}}{2 \tau}
$$

where $\nu$ is the average event rate.

We can also obtain $\sigma^{2}$ with a frequency-domain approach, assuming a white spectral density $S_{\mathrm{W}}$ (two-sided) for the input noise, or

$$
\sigma^{2}=\int_{-\infty}^{\infty} S_{W}|F|^{2} d f=S_{W} \int_{-\infty}^{\infty} \frac{1}{1+\omega^{2} \tau^{2}} d f=\frac{S_{W}}{2 \tau} .
$$

Comparing (37) and (38) we obtain $S_{\mathrm{W}}=\nu A^{2}$, as expected from Carson's theorem. The fact that the input noise is white is rather intuitive. In fact the correlation function apparently tends to vanish anywhere except that in the origin. This yields noise whiteness. 


\section{ACKNOWLEDGMENT}

The authors would like to thank T. Kröll for providing the noise signals acquired with the MARS HPGe detector. The authors would also thank M. Maderna for help in developing the software procedure.

\section{REFERENCES}

[1] [Online]. Available: http://agata.pd.infn.it/documents/Agata_proposal. pdf

[2] I. Y. Lee, "Gamma-ray tracking detectors," Nucl. Instrum. Methods, vol. A422, pp. 195-200, 1999.

[3] "LNL Annual Report 2001,", 2001.

[4] T. Kröll and D. Bazzacco, "Simulation and analysis of pulse shapes from highly segmented HPGe detectors for the gamma-ray tracking array MARS," Nucl. Instrum. Methods, vol. A463, pp. 227-249, 2001.
[5] E. Gatti, A. Geraci, G. Casati, S. Riboldi, and G. Ripamonti, "Spatial localization of multiple simultaneous hits in segmented HPGe detectors: a new algorithm," Nucl. Instrum. Methods, vol. A458, no. 3, pp. 738-744, 2001.

[6] A. Pullia, "How to derive the optimum filter in presence of arbitrary noises, time-domain constraints, and shaped input signals: a new method," Nucl. Instrum. Methods, vol. 397, pp. 414-425, 1997.

[7] K. B. Oldham and J. Spanier, The Fractional Calculus. New York: Academic, 1974

[8] V. Radeka, "1/|f| noise in physical measurements," IEEE Trans. Nucl. Sci. , vol. NS-16, pp. 17-35, Oct. 1969.

[9] A. Pullia, "Impact of nonwhite noises in pulse amplitude measurements: a time domain approach," Nucl. Instrum. Methods, vol. A405, pp. $121-125,1998$.

[10] E. Gatti, P. F. Manfredi, M. Sampietro, and V. Speziali, "Suboptimal filtering of $1 / \mathrm{f}$ noise in detector charge measurements," Nucl. Instrum. Methods, vol. A297, pp. 467-478, 1990.

[11] A. Van Der Ziel, "Noise in solid-state devices and lasers," Proc. IEEE, vol. 58, pp. 1178-1206, Aug. 1970 